\title{
Study of the role of L-arginine in the diagnosis of pregnancy-induced hypertension
}

\author{
Agnieszka Grafka', Maciej Łopucki', Katarzyna Karwasik-Kajszczarek², Marzena Stasiak-Kosarzycka², \\ Andrzej Miturski', Grzegorz Dzida ${ }^{4}$ \\ 'I Chair and Department of Oncological Gynaecology and Gynaecology, Medical University of Lublin, Poland \\ ${ }^{2}$ Chair and Department of Obstetrics and Pathology of Pregnancy, Medical University of Lublin, Poland \\ ${ }^{3}$ Department of Gynaecology and Obstetrics with Admission Room of the Cardinal Wyszyński Regional Specialist Hospital in Lublin, Poland \\ ${ }^{4}$ Department of Internal Diseases, Medical University of Lublin, Poland
}

This study was supported by Grant of Medical University of Lublin, Poland (MNsd127).

\begin{abstract}
Background. Pregnancy-induced hypertension (PIH) is a significant health issue in pregnancy, complicating 7-10\% of pregnancies. L-arginine is an important mediator of vasodilation with a potential preventative role in pregnancy-related hypertensive diseases.

Aim of the study. The aim of the present study was to assess the differences in plasma L-arginine concentrations in physiological pregnancies and pregnancies complicated with hypertension in the third trimester.

Material and methods. Plasma concentration of L-arginine was determined by ion-exchange chromatography in 210 pregnant women (25-41 weeks of gestation). Plasma L-arginine concentration was expressed in $\mu \mathrm{mol} / \mathrm{cm}^{3}$.

Results. The mean L-arginine concentration was significantly higher in physiological pregnancy $(0.102)$ than in the PIH group (0.034). The analysis of plasma L-arginine concentration in the subgroups of third trimester showed that L-arginine concentration in the PIH group decreased with increasing stage of pregnancy (25-34 hbd - 0.051 ; $35-38 \mathrm{hbd}-0.03$; 39-41 hbd - 0.02). L-arginine concentration in physiological pregnancies was the same in all subgroups (0.1).

Conclusions. L-arginine may have a role in the prevention and treatment of pregnancy-induced hypertension. Further well-designed and adequately powered research is warranted.

key words: L-arginine, pregnant women, hypertension, blood plasma

Arterial Hypertens. 2016, vol. 20, no. 3, pages: 113-118

DOI: 10.5603/AH.2016.0017
\end{abstract}

\section{Introduction}

Pregnancy-induced hypertension (PIH) is a pregnancy-specific hypertensive disorder, which can complicate $7-10 \%$ of pregnancies $[1,2]$. The pathophysiology of hypertension and proteinuria in pregnancy, known as PIH, remains largely undiscovered. Hypertensive disorders of pregnancy contribute signifi-

Address for correspondence: dr n. med. Agnieszka Grafka I Chair and Department of Oncological Gynaecology and Gynaecology Medical University of Lublin

Staszica 16, 20-081 Lublin, Poland

Phone: +4881 5327847; fax: +48815320608

E-mail: grafkaagnieszka@yahoo.com

VM Copyright (c) 2016 Via Medica, ISSN 2449-6170 cantly to both perinatal and maternal mortality and morbidity on global scale [3-5]. More than 30 years ago, Dr. Leon Chesley, an expert in the field of hypertension in pregnancy, divided the most likely causative factors into four major categories: placental, renal, immunologic and dietary [6]. According to Saczko et al. [1], one of the PIH causes is excessive production of free radicals that induce arterial vasoconstriction. The other factors predisposing pregnant women to $\mathrm{PIH}$ include multiple pregnancy, foetal male gender, young maternal age (under 18 years of age in primiparas), advanced maternal age (over 40 years of age in multiparas), obesity, genetic load, chronic stress and excess sodium in diet [3-5]. However, PIH also oc- 
curs in pregnant women without the above-mentioned factors. Therefore, Skoczyńska et al. [2] believe that further research on this subject is needed.

$\mathrm{PIH}$ is one of the most serious obstetric complications. Despite intensive studies carried out in many centres worldwide, the aetiopathogenesis of PIH has not been explained. It is evident that there is abnormal placentation and defective trophoblast invasion resulting in the utero-placental unit being under perfused [1]. These phenomena are associated with ischaemia and subsequent hypoxia leading to endothelial damage and production of vasoactive factors, which promote vasoconstriction. In response to that, nitric oxide is synthesized from the amino acid L-arginine $[1,7]$.

Under physiological conditions, endothelial cells secrete a number of active metabolites, which indirectly or directly affect proper vascular contraction and relaxation. This leads to imbalance, predominantly in the prostacyclin and nitric oxide/thromboxane and endothelin system [8]. In pregnant women with PIH, an increase in thromboxane concentration $\left(\mathrm{TXA}_{2}\right.$, Thromboxane $\mathrm{A}_{2}$ ) in relation to prostacyclin $\left(\mathrm{PGI}_{2}\right.$, Prostacyclin) is observed, which induces increased vasoconstriction, damage to and aggregation of blood platelets as well as reduced blood flow via the spiral arteries. Physiologically, prostacyclin is a vasodilator and its high concentration determines proper trophoblast invasion into the walls of spiral arteries. Thromboxane, being its antagonist, strongly contracts vessels and increases platelet aggregation $[8,9]$.

Nitric oxide (NO) has a potent vasodilator effect, which is involved in inhibition of thromboxane production, platelet aggregation and stimulation of prostacyclin production [10-12]. Thanks to its properties, it plays a significant role in obstetric procedures like delivery, cervical ripening, intrauterine growth retardation and occurrence of PIH [13]. During pregnancy, the blood vessels undergo adaptive changes due to blood volume gain, reduced vascular tolerance and increased cardiac output, which lead to an increase in $\mathrm{NO}$ production. The most important $\mathrm{NO}$ donor is L-arginine. In the absence of L-arginine, gestational hypertension develops. Facchinetti et al. [14] have demonstrated that L-arginine supplementation could significantly decrease blood pressure in PIH women.

Arginine (2-amino-5-guanidinopentanoic acid; L-ARG) is a semi-essential amino acid, physiologically active in its L-form, which is synthesized by endothelial cells and excreted with urine in vivo [15]. L-arginine has been well recognized as a drug to treat variable diseases and symptoms, as well as a dietary tonic. $\mathrm{L}$-arginine is the substrate of $\mathrm{NO}$, a potent vasodilator, which may play a major role in regulating blood pressure [16]. Scibor and Czeczot [15] and Wu et al. [17] believe that the main sources of L-arginine in the body are food, intracellular protein degradation and protein endogenous synthesis. An adult consumes about $5.4 \mathrm{~g}$ of L-arginine a day [18, 19], and about $50 \%$ of dietary L-arginine gets to the cardiovascular system $[18,20]$. Moreover, 5 to $15 \%$ of plasma L-arginine in adults is endogenously synthesized from citrulline. The total plasma concentration of L-arginine depends on the developmental stage of an individual and diet, and ranges from 95 to $250 \mu \mathrm{mol} / 1[19,20]$.

Scibor and Czeczot [15] have demonstrated that $\mathrm{L}$-arginine is used for the synthesis of proteins, urea, creatinine, proline, polyamines and NO. It is metabolized to ornithine and urea in the urea cycle in the liver $[18,21]$. In the kidneys and pancreas, it is a substrate in the synthesis of guanidoacetate, which is subsequently converted into creatinine $[20,21]$. Moreover, L-arginine plays a special role in the cardiovascular system, where as a source of NO it affects vasodilation. Continuous synthesis of NO in the endothelium prevents the development of hypertension and other vascular diseases [15, 22]. Relaxation of vascular smooth muscles in response to $\mathrm{NO}$ effects is associated with the activity of cGK I $\alpha$ (a protein kinase $\mathrm{G}$ isoform) and the muscle tone is regulated by phosphorylation of the myosin light chain. The final stage of $\mathrm{NO}$ action is lack of phosphorylation of the myosin light chain leading to relaxation of vascular smooth muscles [23, 24].

Wu and Meininger [25] as well as Wu and Morris [20] believe that the major source of $\mathrm{L}$-arginine for $\mathrm{NO}$ synthesis in the vascular endothelial cells is extracellular L-arginine in blood plasma whose $54 \%$ is used for the synthesis of NO; the remaining amount originates from endogenous sources. Released from the vascular endothelium, NO diffuses not only to the muscles but also to the lumen of blood vessels where it binds haemoglobin in the erythrocytes. The findings reported by Davisa et al. [26] and Moncada et al. [27] indicate that haemoglobin can be one of the crucial stores of NO in the body. Further studies on this issue are likely to confirm that NO release from haemoglobin is possible, which would suggest that NO can act longer than several seconds and not only at the site of its synthesis [26, 27].

Administration of L-arginine seems to improve uterine-placental circulation and decrease maternal blood pressure [28]. Therefore, L-arginine supplementation might be a new therapeutic option and its plasma level a new diagnostic option in quick diagnosis and appropriate treatment of PIH. 


\section{Objective}

The aim of the present study was to assess the differences in plasma L-arginine concentrations in physiological pregnancies and pregnancies complicated with hypertension in the third trimester.

\section{Material and methods}

\section{Patients}

The study involved 210 pregnant women, 25-41 weeks of gestation, managed in the Chair and Department of Obstetrics and Pathology of Pregnancy, Medical University of Lublin and Department of Gynaecology and Obstetrics with Admission Room of the Cardinal Wyszyński Regional Specialist Hospital in Lublin in the years 2010-2014. The overall number of women was divided into 2 groups: study group and control group.

Women in the control group $(\mathrm{n}=105)$ did not take any drugs that could affect the cardiovascular system and had blood pressure within normal limits $(120 / 80 \mathrm{~mm} \mathrm{Hg})$. The mean age of women was 28.6 years, height $-165.93 \mathrm{~cm}$, body weight -84.11 $\mathrm{kg}$, systolic blood pressure (SBP $-119.54 \mathrm{~mm} \mathrm{Hg}$ and diastolic blood pressure (DBP) $-77.17 \mathrm{~mm} \mathrm{Hg}$. Women in the study group $(\mathrm{n}=105)$ had elevated blood pressure (> 140/90 mm Hg) and no concomitant diseases that could increase blood pressure (e.g. diabetes). The mean age in this group was 32.11 years, height $-163.01 \mathrm{~cm}$, body weight $-96.48 \mathrm{~kg}$, SBP $-162.98 \mathrm{~mm} \mathrm{Hg}$ and DBP $-97.67 \mathrm{~mm} \mathrm{Hg}$. According to the recommendations of the European Society of Hypertension (ESH) and the European Society of Cardiology (ESC), blood pressure was measured twice at a 6-hour interval in a sitting position using an electronic sphygmomanometer [29, 30]. To obtain detailed data, women in the study and control groups were divided into 3 subgroups: 25-34, 35-38, 39-41 hbd, 35 cases each.

Experimental procedures were approved by the Bioethical Commission of the Medical University of Lublin, Poland (No KE-0254/223/2010). All women accepted the study protocol and gave written informed consent for participation.

\section{Biochemical analyses}

The study material was venous blood collected in the fasting state from women with physiological pregnancies and pregnancies complicated with hypertension, $9 \mathrm{ml}$, to the test-tubes containing lithium heparin (aspiration-vacuum systems, Sarstedt, Germany).
The plasma from each blood sample was collected immediately after centrifugation at $4000 \times \mathrm{g}$ for $10 \mathrm{~min}$ and then stored at $-80^{\circ} \mathrm{C}$ until analysis. For $\mathrm{L}$-arginine concentration measurements, plasma was deproteinised with $6 \%$ sulphosalicylic acid in lithium-citrates buffer $(\mathrm{pH}=2.6)$ and centrifuged at $12000 \times \mathrm{g}$ for $20 \mathrm{~min}$. The obtained plasma was used for $\mathrm{L}$-arginine determination in an ion-exchange chromatography (INGOSAAA-400 apparatus for automatic analysis of amino acids, Ingos Corp., Prague, Czech Republic). Amino acids were separated using the analytical column OSTION LG FA and identified in comparison to the standards provided by INGOS Corp. The original software MIKRO version 1.8.0 (INGOS) was used for amino acid determinations. All the tests were performed in duplicate.

Plasma L-arginine concentration was expressed in $\mu \mathrm{mol} / \mathrm{cm}^{3}$.

\section{Statistical analysis}

Statistical analyses were performed using Statistica v. 10.0 software (StatSoft, USA). Data were presented as a mean and standard deviation (SD). The distribution of quantitative parameters was assessed using the Shapiro-Wilk W test. All quantitative parameters were characterised by skew distribution; therefore, non-parametric tests were applied to evaluate inter-subgroup significant differences. Two independent groups were compared using the Mann-Whitney $\mathrm{U}$ test. Inter-quantitative parameter correlations were assessed by Spearman's rank correlation.

The differences between mean values were considered as statistically significant at $\mathrm{p}<0.05$.

\section{Results}

The mean L-arginine concentration was significantly higher in the control group of pregnancy than in PIH group. Results are presented in Table I and Figure 1.

Analysis of L-arginine plasma concentration in the subgroups of third trimester showed that L-arginine concentration in the PIH group decreased with increasing stage of pregnancy (Fig. 2). L-arginine concentration in control pregnancies was the same in all subgroups $-0.1 \mu \mathrm{mol} / \mathrm{cm}^{3}$.

Analysis of correlations between L-arginine concentration, systolic and diastolic pressure in PIH-complicated pregnancies demonstrated a weak association between L-arginine concentration and SBP (Tab. II). 
Table I. Plasma concentration of L-arginine in the control and PIH group in the third trimester of pregnancy

\begin{tabular}{|l|c|c|c|}
\hline \multirow{2}{*}{ Parameter } & \multicolumn{2}{|c|}{ Mean \pm SD } & \multirow{2}{*}{$P$ value } \\
\cline { 2 - 4 } & Control group $(\mathbf{n}=105)$ & PIH group $(\mathbf{n}=105)$ & $<0.00005$ \\
\hline L-ARG $\left[\mu \mathrm{mol} / \mathbf{c m}^{3}\right]$ & $0.102 \pm 0.013$ & $0.034 \pm 0.014$ & \\
\hline
\end{tabular}

Table II. Correlation between plasma L-arginine concentration in the study group versus systolic and diastolic pressure

\begin{tabular}{|l|c|c|l|c|c|}
\hline \multicolumn{7}{|c|}{ PIH group ( $\mathbf{n}=105)$} \\
\hline $\begin{array}{l}\text { L-ARG } \\
\text { \& SBP }\end{array}$ & $\mathbf{R}$ & $\mathbf{P}$ & & $\mathbf{R}$ & $\mathbf{P}$ \\
\hline
\end{tabular}

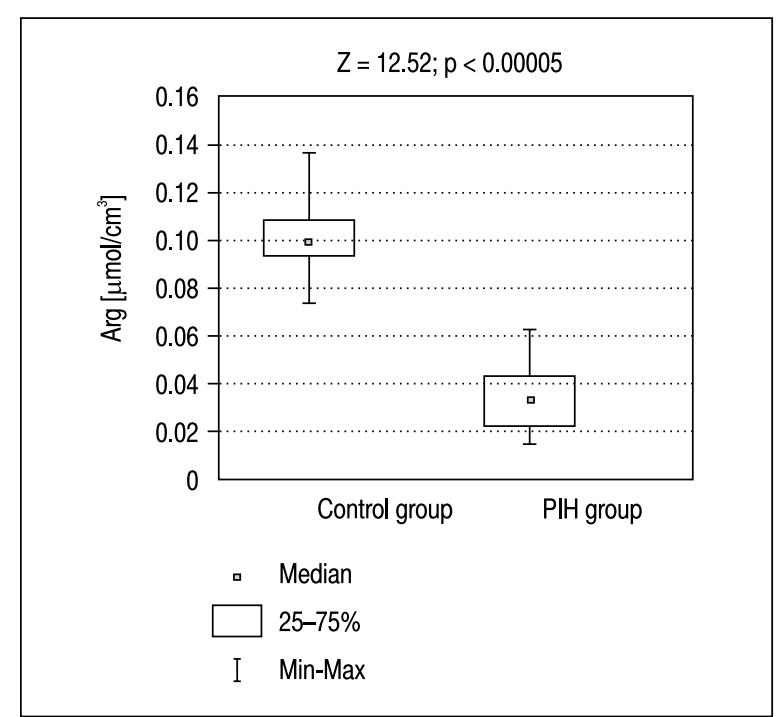

Figure 1. Plasma concentration of L-arginine in the control and PIH group in the third trimester of pregnancy

\section{Discussion}

It is well known that hypertensive disorders in pregnancy are an important cause of maternal and neonatal mortality. The main features in $\mathrm{PIH}$ include developing systolic blood pressure (SBP) $\geq 140$, or diastolic blood pressure $(\mathrm{DBP}) \geq 90$, and proteinuria of 0.3 grams or greater in a 24-hour urine specimen after 20 weeks of gestation in a woman who was previously normotensive. Hypertension is generally the earliest physical abnormality seen in PIH and is the most important clinical clue to the presence of diseases [31].

Cnossen et al. [32] have published a meta-analysis in which different indices of blood pressure to predict PIH have been comprehensively evaluated. This meta-analysis included 34 studies and evaluated SBP, DBP, mean arterial pressure (MAP), and the increase over time in blood pressure. The data from this meta-analysis support the conclusion that blood pressure measurements in the first and second

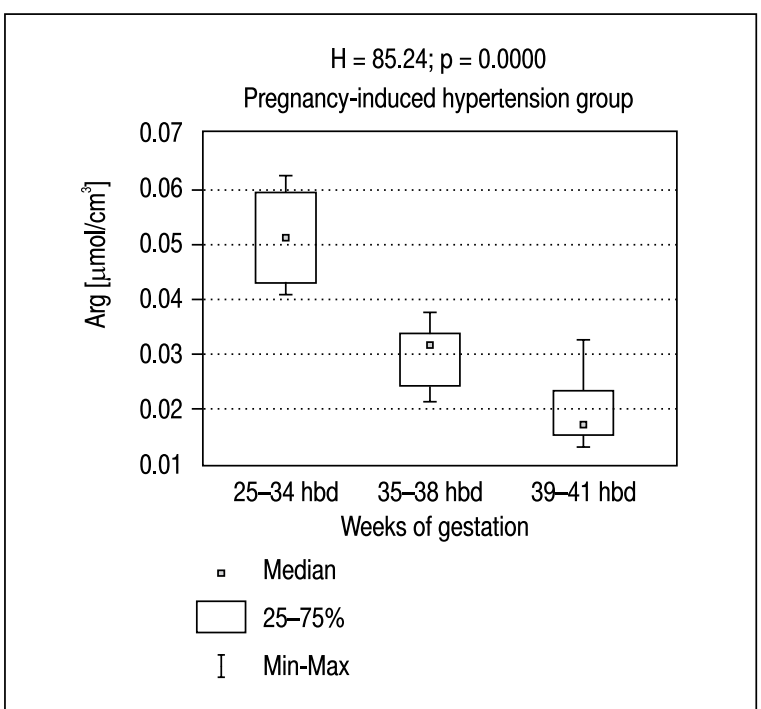

Figure 2. L-arginine plasma concentration in the PIH group, in the subgroups of third trimester

trimester have only a modest ability to predict hypertensive disorders [32]. Therefore, in our research we focused our attention only on the third trimester of pregnancy.

L-arginine is a semi-essential amino acid, and during pregnancy, under the circumstances of increased nitric oxide production, endogenous synthesis is insufficient [33]. L-arginine concentrations have been demonstrated to be significantly reduced in women with PIH when compared with healthy women without the disease $[34,35]$.

The results of our study indicate that L-arginine might be a new diagnostic factor for pregnant women, which can help in quick diagnosis. Occurrence of PIH is associated with a significant reduction in plasma L-arginine concentration. The lower the concentration, the more advanced the pregnancy is. Although L-arginine may represent a promising diagnostic factor, there is currently limited information available to assess its diagnostic utility. 
Intracellular protein distribution induces the release of free amino acids, which are precursors for synthesis of other proteins. The intracellular pool of amino acids is in balance with the extracellular pool in plasma. This balance is regulated by membranous transport characteristic of individual amino acids. Another source of extracellular pool is amino acids derived from dietary proteins [36].

L-arginine is a nutrient essential for foetal life and growth [37]. Battaglia et al. [38] have demonstrated that its oral administration ( $16 \mathrm{~g} /$ daily for 8 days) can enhance the uterine blood flow and ovarian response to gonadotropin, have beneficial effects on the endometrium and increase the fertility of women with conception-related problems after in vitro procedures. Based on the findings of animal studies, numerous clinical trials were performed evaluating the effects of L-arginine on improvement of fertility and prevention of intrauterine growth restriction (IUGR) in pregnant women. Xiao and Li [39] have shown that daily intravenous infusion of L-arginine ( $20 \mathrm{~g}$ a day for 7 days) at the 33 week of pregnancy complicated by IUGR of unknown causes increased the foetal birth weight by $6.4 \%$. Oral and intravenous L-arginine supplementation involving healthy volunteers and pregnant women appears to be well tolerated, with no significant adverse effects, even in doses as high as $20 \mathrm{~g}$ daily [40, 41]. Lampariello et al. [42], who administered oral L-arginine ( $6 \mathrm{~g} /$ day) to 43 pregnant women with IUGR, have observed significant clinical improvement in 32 cases and complete recovery in 19 pregnant women. Moreover, studies by Shen and $\mathrm{Hu}$ [43] have disclosed that L-arginine administered to women with IUGR decreases apoptosis in the placenta and substantially improves the foetal growth and development. A meta-analysis of the data of seven clinical studies involving 916 patients has demonstrated that L-arginine supplementation can reduce diastolic blood pressure and prolong the physiological pregnancy and pregnancy complicated by hypertension with or without proteinuria [44]. Based on the above findings, L-arginine can be considered to have beneficial effects on blood pressure regulation and can be used for quick diagnosis of pregnant women as well as prompt institution of appropriate treatment, including L-arginine supplementation. Our study showed a weak negative correlation between L-arginine concentration and SBP but not DBP. Literature data revealed that L-arginine could decrease DBP and SBP; however, a decline in SBP was not statistically significant [45]. It is possible because the high L-arginine leads to high nitric oxide synthesis resulting in blood pressure lowering and inversely.
Evidence specifically relating to the mechanistic actions of $\mathrm{L}$-arginine in PIH is more limited. L-arginine is the most important NO donor [14]. Davidge et al. [46] estimated nitrate concentrations in women with $\mathrm{PIH}$, indicating a reduction in the $\mathrm{NO}$ production compared with healthy pregnant women.

Our findings show that plasma L-arginine concentration is lower in the group with higher blood pressure compared with physiological pregnancies. These results are confirmed by Kim et al. [34] and D'Aniello et al. [47].

Endothelial dysfunctions associated with impaired synthesis of NO are considered one of the causes of PIH. Cooke et al. [48] and Wu and Meininger [49] have found that administration of L-arginine to pregnant women increased the production of $\mathrm{NO}$ in the peripheral vessels and reduced blood pressure. Moreover, Wu and Meininger [49] and Wu et al. [19] have observed good outcomes using L-arginine for the treatment of arterial hypertension, hypertension-related pregnancy pathologies, ischaemic diseases, circulatory failure, atherosclerosis, diabetes mellitus, glaucoma, cerebral stroke, vascular thrombosis, renal failure, liver dysfunction with urea cycle disorders and ammonia intoxications. L-arginine administered to women with PIH in the doses of $6 \mathrm{~g} /$ day normalised arterial blood pressure [50].

\section{Conclusions}

There is currently limited unequivocal information available in literature about the evaluation of $\mathrm{L}$-arginine levels in PIH diagnosis. The literature data give grounds for clinical use of L-arginine in prophylaxis and monitoring of metabolic diseases and arterial hypertension whereas our findings indicate that quick detection of decreased plasma concentrations of L-arginine should enable earlier institution of appropriate treatment of pregnant women, can prevent the development of fully symptomatic PIH or alleviate its course. This should improve the comfort of life and favourably affect proper foetal development. These benefits ought to be confirmed by larger and more-powered studies.

\section{References}

1. Saczko Z., Saczko J., Kulbacka J., Chwiłkowska A., Żórawski K. Pregnancy induced hypertension. Etiopathogenesis. Arterial Hypertens. 2009; 13: 199-205.

2. Skoczyńska A., Turczyn B., Murawski M., Wojakowska A., Wenecka B. Arterial hypertension in pregnant women in relation to professional work. Arterial Hypertens. 2011; 15: 290-298. 
3. Ananth C.V., Savitz D.A., Bowes W.A.Jr. Hypertensive disorders of pregnancy and stillbirth in North Carolina, 1988 to 1991. Acta Obstet Gynecol Scand. 1995; 74: 788-793.

4. Sibai B.M., Hauth J., Caritis S. et al. Hypertensive disorders in twin versus singleton gestations. National Institute of Child Health and Human Development Network of Maternal-Fetal Medicine Units. Am. J. Obstet. Gynecol. 2000; 182: 938-942.

5. Solomon G.C., Carroll J.S., Okamura K., Graves S.W., Seely EW. Higher cholesterol and insulin levels in pregnancy are associated with increased risk for pregnancy-induced hypertension. Am. J. Hypertens. 1999; 12: 276-282.

6. Chesley L.C. Hypothesis. Hypertensive Disorders in Pregnancy. Appleton-Century Crofts. New York, 1978.

7. Roberts J.M., Carl A., Hubel A. Oxidative stress in preeclampsia. Am. J. Obstet. Gyneacol. 2004; 190: 1177-1178.

8. Dietl J. The pathogenesis of pre-eclampsia: new aspects. J. Perinat. Med. 2000; 28: 464-471.

9. Walsh S.W. Eicosanoids in preeclampsia, Prostaglandins. Leukot. Essent. Fatty Acids 2004; 70: 223-232.

10. Benyó Z., Görlach C., Wahl M. Involvement of thromboxane A2 in the mediation of the contractile effect induced by inhibition of nitric oxide synthesis in isolated rat middle cerebral arteries. J. Cereb. Blood Flow Metab. 1998; 18: 616-618.

11. Wang W., Diamond S.L. Does elevated nitric oxide production enhance the release of prostacyclin from shear stressed aortic endothelial cells? Biochem. Biophys. Res. Commun. 1997; 233: 748-751.

12. Riddell D.R., Owen J.S. Nitric oxide and platelet aggregation. Vitam. Horm. 1999; 57: 25-48.

13. Rees D.D., Palmer R.M., Moncada S. Role of endotheliumderived nitric oxide in the regulation of blood pressure. Proc. Natl. Acad. Sci. USA 1989; 86: 3375-3378.

14. Facchinetti F., Longo M., Piccinini F., Neri I., Volpe A. L-arginine infusion reduces blood pressure in preeclamptic women through nitric oxide release. J. Soc. Gynecol. Invest. 1999; 6: 202-207.

15. Ścibor D., Czeczot H. Arginine - Metabolism and Functions in Cardiovascular System. Adv. Clin. Exp. Med. 2005; 14: 1041-1050.

16. Palmer R.M., Ashton D.S., Moncada S. Vascular endothelial cells synthesize nitric oxide from L-arginine. Nature 1988; 333: 664-666.

17. Wu G., Wu Z., Dai Z. et al. Dietary requirements of "nutritionally non-essential amino acids" by animals and humans. Amino Acids 2013; 44: 1107-1113.

18. Ścibor D., Czeczot H. Arginine - metabolism and functions in the human organism. Postepy Hig. Med. Dosw. 2004; 58: 321-332.

19. Wu G., Meininger C.J., Knabe D.A., Bazer F.W., Rhoads J.M. Arginine nutrition in development, health and disease. Curr. Opin. Clin. Nutr. Metab. Care 2000; 3: 59-66.

20. Wu G., Morris S.M.Jr. Arginine metabolism: nitric oxide and beyond. Biochem. J. 1998; 336: 1-17.

21. Blantz R.C., Satriano J., Gabbai F., Kelly C. Biological effects of arginine metabolites. Acta Physiol. Scand. 2000; 168: 21-25.

22. Gornik H.L., Creager M.A. Arginine and endothelial and vascular health. J Nutr. 2004; 134 (10 Suppl.): 2880S-2887S.

23. Fischmeister R., Mery P.F. Regulation of cardiac calcium channels by cGMP and NO. In: Morad M., Ebashi S., Trautwein W., Karachi U. (eds.). Molecular Physiology and Pharmacology of Cardiac Ion Channels and Transporters. Kluwer Academic Publishers, London 1997; 93-105.

24. Sawada N., Itoh H., Yamashita J. et al. cGMP dependent protein kinase phosphorylates and inactivates RhoA. Biochem. Biophys. Res. Commun. 2001; 280: 798-805.

25. Wu G., Meininger C.J. Regulation of nitric oxide synthesis by dietary factors. Annu. Rev. Nutr. 2002; 22: 61-86.

26. Davis K.L., Martin E., Turko I.V., Murad F. Novel effects of nitric oxide. Annu. Rev. Pharmacol. Toxicol. 2001; 41: 203-236.

27. Moncada S., Palmer R.M., Higgs E.A. Nitric oxide: physiology, pathophysiology and pharmacology. Pharmacol. Rev. 1991; 43: 109-142.
28. Neri I., Valensise H., Facchinetti F., Menghini S., Romanini C., Volpe A. 24-hour ambulatory blood pressure monitoring: A comparison between transdermal glyceryl-trinitrate and oral nifedipine. Hypertens. Pregnancy 1999; 18: 107-113.

29. Brown M.A., Whitworth J.A. Management of hypertension in pregnancy. Clin. Exp. Hypertens. 1999; 21: 907-916.

30. Burlew B.S., Horn H.R., Sullivan J.M. Pregnancy and the heart. In: Diagnostic atlas of the heart. (eds.). Hurst J.W., Alpert J.S. Raven Press, New York 1994; 535-544.

31. Rapacz A., Filipek B. Hypertension in pregnancy. Farm. Pol. 2009; 65: 581-585.

32. Cnossen J.S., Vollebregt K.C., De Vrieze N. et al. Accuracy of mean arterial pressure and blood pressure measurements in predicting pre-eclampsia: systematic review and meta-analysis. BMJ 2008; 336: 1117-1120.

33. Morris N.H., Eaton B.M., Dekker G. Nitric oxide, the endothelium, pregnancy and preeclampsia. BJOG 1996; 103: 4-15.

34. Kim Y.J., Park H.S., Lee H.Y. et al. Reduced L-arginine level and decreased placental eNOS activity in preeclampsia. Placenta 2006; 27: 438-444.

35. Neri I., Piccinini F., Marietta M., Facchinetti F., Volpe A. Platelet responsiveness to $\mathrm{L}$-arginine in hypertensive disorders of pregnancy. Hypertens. Pregnancy 2000; 19: 323-330.

36. Kalhan S.C. Protein metabolism in pregnancy. Am. J. Clin. Nutr. 2000; 71 (5 Suppl): 1249S-1255S.

37. Wu G., Bazer F.W., Satterfield M.C. et al. Impacts of arginine nutrition on embryonic and fetal development in mammals. Amino. Acids 2013; 45: 241-256.

38. Battaglia C., Salvatori M., Maxia N., Petraglia F., Facchinetti F., Volpe A. Adjuvant L-arginine treatment for in-vitro fertilization in poor responder patients. Hum. Reprod. 1999; 14: 1690-1697.

39. Xiao X.M., Li L.P. L-Arginine treatment for asymmetric fetal growth restriction. Int. J. Gynaecol. Obstet. 2005; 88: 15-18.

40. Neri I., Mazza V., Galassi M.C., Volpe A., Facchinetti F. Effects of L-arginine on uteroplacental circulation in growth-retarded fetuses. Acta Obstet. Gynecol. Scand. 1996; 75: 208-212.

41. Facchinetti F., Neri I., Genazzani A.R. L-arginine infusion reduces preterm uterine contractions. J. Perinat. Med. 1996; 24: 283-285.

42. Lampariello C., De Blasio A., Merenda A., Graziano E., Michalopoulou A., Bruno P. Use of arginine in intrauterine growth retardation (IUGR). Author's experience. Minerva Ginecol. 1997; 49: 577-581.

43. Shen S.F., Hua C.H. Effect of L-arginine on the expression of Bcl-2 and Bax in the placenta of fetal growth restriction. J. Matern. Fetal. Neonatal. Med. 2011; 24: 822-826.

44. Gui S., Jia J., Niu X. et al. Arginine supplementation for improving maternal and neonatal outcomes in hypertensive disorder of pregnancy: A systematic review. J. Renin Angiotensin Aldosterone Syst. 2014; 15: 88-96.

45. Orozco-Gutiérrez J.J., Castillo-Martínez L., Orea-Tejeda A. et al. Effect of L-arginine or L-citrulline oral supplementation on blood pressure and right ventricular function in heart failure patients with preserved ejection fraction. Cardiol. J. 2010; 17: 612-618.

46. Davidge S.T., Stranko C.P., Roberts J.M. Urine but not plasma nitric oxide metabolites are decreased in women with preeclampsia. Am. J. Obstet. Gynecol. 1996; 174: 1008-1013.

47. D'Aniello G., Tolino A., Fisher G. Plasma L-arginine is markedly reduced in pregnant women affected by preeclampsia. J. Chromatogr. B. Biomed. Sci. Appl. 2001; 753: 427-431.

48. Cooke J.P., Mont-Reynaud R., Tsao P.S., Maxwell A.J. Nitric oxide and vascular disease. In: Nitric oxide: biology and pathology. (ed.). Ignarro L.J. Academic Press, New York 2000; 759-783.

49. Wu G., Meininger C.J. Arginine nutrition and cardiovascular function. J Nutr. 2000; 130: 2626-2629.

50. Kostka-Trąbka E. Arginine - an amino acid of new opportunities of clinical application. Ordynator Leków 2002; 3: 15-19. 